Ann. Biol. anim. Bioch. Biophys., Ig64, 4 (I), 5-I5.

\title{
HYPOTRICHOSE PÉRIODIQUE CHEZ LA SOURIS. INFLUENCE MATERNELLE : RANG DE LA PARTURITION, FACTEUR SAISON ET AGE DE LA MÈRE
}

\author{
N. KOBOZIEFF, Nathalie POMRIASKINSKY-KOBOZIEFF et E. GEMAHLING. \\ Laboratoire de Génétique, Ecole nationale vétérinaire, Alfort (Seine).
}

\section{SOMMAIRE}

L'hypotrichose périodique chez la souris est une mutation récessive, à récessivité incomplète et à faible pénétrance. D'autres facteurs interviennent dans la fréquence d'apparition de cette anomalie. Cet article met en évidence le rôle que jouent l'influence maternelle et le facteur saison dans la fréquence d'apparition de sujets anormaux. Notre étude a porté sur 256 I souris $\mathrm{C}_{57} \mathrm{BL} / \mathrm{Jax} 6$, dont $22 \mathrm{I} 4$ sujets normaux et 347 atteints d'hypotrichose périodique.

Influence du rang de la parturition : les primipares donnent le plus grand pourcentage de sujets anormaux, les bipares fournissent un pourcentage nettement inférieur et nous n'avons observé aucun sujet anormal dans les portées des multipares.

L'influence du facteur saison est très nette. Les expériences de croisements se poursuivent sur toute l'année, or nous avons observé que le plus grand nombre de sujets atteints apparaît parmi les naissances qui se situent entre les mois de juin et d'août.

Influence de l'âge de la mère : parmi les primipares ce sont les mères les plus jeunes (jusqu'à 70 jours), qui fournissent le plus grand pourcentage de sujets atteints, ce pourcentage baisse dans les portées des mères d'âge moyen pour remonter dans celles des mères âgées de plus de r6 I jours.

Il existe un rapport entre l'influence de l'âge de la mère et le facteur saison : nous constatons que les primipares très jeunes semblent échapper à l'influence du facteur saison qui devient de plus en plus frappante au fur et à mesure que l'âge de la mère augmente. C'est entre les mois de mars à août que l'on observe le plus grand pourcentage de sujets atteints. Il en est de même pour les bipares. Les mères les plus âgées sont nettement plus sensibles au facteur saison.

\section{IN'TRODUC'TION}

Dans un article précédent, nous avons décrit l'évolution des mues anormales chez les souris atteintes d'hypotrichose périodique (épilation entre le $16^{\mathrm{e}}$ et le $24^{\mathrm{e}}$ jour après la naissance : 6 types différents). Nous avons assisté à plusieurs épilations concordant, plus ou moins, avec les mues normales. Nous avons établi que l'hypo- 
trichose périodique est une mutation récessive, à récessivité incomplète et à très faible pénétrance; nous avons relevé la présence de sujets " normal overlaps", c'est-à-dire de sujets phénotypiquement normaux se comportant génotypiquement comme des anormaux.

De toutes les anomalies du poil relevées dans la littérature, seule celle décrite par LOEFFLER est phénotypiquement semblable à la nôtre. Du point de vue génétique, notre mutation est différente de toutes celles décrites y compris celle étudiée par LOEFFLER.

Dans le présent article nous cherchons à établir le rôle exact joué par l'âge de la mère dans la fréquence d'apparition de l'anomalie et son influence sur les facteurs "rang de parturition " et "saison".

De nombreux auteurs ont fait ressortir l'influence maternelle dans la fréquence d'apparition de diverses anomalies dans les différentes espèces.

WRIGHT (I934) a constaté que, dans les lignées consanguines de cobayes atteints de polydactylie, la fréquence d'apparition de sujets anormaux varie suivant l'âge de la mère, les pourcentages de sujets anormaux les plus importants sont observés dans les portées des mères les plus jeunes. HoLr (I948), signale le même phénonìne dans les croisements d'homozygotes des lignées de souris polydactyles. GrEEN et RUSSELL (I95I) ont observé une variation dans le nombre des vertèbres lombaires et sacrées chez les souris albinos de Bagg; là aussi les mères les plus jeunes fournissent le plus grand nombre de sujets atteints. SEARLE (I954), a démontré que l'âge de la mère joue un rôle important dans la fréquence d'apparition de différentes anomalies du squelette telles que : malformation des vertèbres (cervicales et thoraciques), malformations du crâne, dés dents et du bassin. Il a, lui aussi, souligné que les femelles les plus jeunes donnent un plus grand nombre de sujets anormaux. Hauschka et Brown (I954) ont étudié des lignées de souris présentant une ouverture palpébrale à la naissance et eux aussi ont observé que la fréquence d'apparition de sujets anormaux dans la lignée $\mathrm{A} / \mathrm{He}$ décline avec le rang de parturition $\left(\sigma^{x} \sigma^{x} I 6,66 \mathrm{p}\right.$. Ioo dans la première portée et seulement $5,06 \mathrm{p}$. Ioo pour les $6^{\mathrm{e}}, 7^{\mathrm{e}}$ et $8^{\mathrm{e}}$ portées ; pour les $\not \varnothing$ il en est de même : 6,52 p. Ioo dans la première portée et seulement $2 \mathrm{p}$. Ioo dans les $6^{\mathrm{e}}, 7^{\mathrm{e}}$ et $8^{\mathrm{e}}$ portées). KoboziefF et PomRIASKINSKY-KoboziefF (I946) à l'occasion d'une étude sur la troncature de l'oreille chez la souris, ont signalé que l'âge de la mère au moment de la mise bas, joue un tôle certain : avec l'augmentation de l'âge de la mère le pourcentage de sujets atteints passe de 36,6 à 23,08 p. Ioo. De nombreuses études ont démontré l'importance pré et post-natale de l'influence maternelle sur le poids des descendants et leur nombre par portée (VENGE I950, BATEMAN I954-I963, ChaI I956, Brumby ig6o et Fax,CONER Ig60).

\section{MATÉRIEL ET TECHNIQUES}

Pour l'étude du rôle du rang de parturition, du facteur saison et de l'influence de l'âge de la mère sur ces deux facteurs, nous avons utilisé le même matériel que pour l'article précédent, à l'exception de deux expériences de croisements ne fournissant pas de sujets anormaux : l'expérience I $(\mathrm{An} \times \mathrm{N})$ et l'expérience $\mathrm{V}(\mathrm{FbN}$ du II $\times \mathrm{FbN}$ du II) ; le tout réparti en 4 séries. 
Série I, comprend 3 expériences effectuées à l'aide de souris de la première génération $\left(\mathrm{F}_{1} \mathrm{~N}\right)$ ou issues du croisement de retour $(\mathrm{FbN})$ :

expérience II : $\delta \mathrm{An} \times$ o $\mathrm{F}_{1} \mathrm{~N}$ issue du croisement $\mathrm{I}(\mathrm{An} \times \mathrm{N})$, ou $\approx \mathrm{F}_{1} \mathrm{~N} \times$ o $\mathrm{An}$;

expérience III : $a^{*}$ An $\times$ o $\mathrm{FbN}$ issue du II ;

expérience $I V: \delta F_{1} N$ issu du $\mathrm{I} \times \stackrel{q}{*} \mathrm{~F}_{1} \mathrm{~N}$ issue du $\mathrm{I}$.

Série $I I$, comprend 2 expériences dans lesquelles interviennent les $q$ a anormales croisées soit avec des $\sigma^{\alpha} \delta$ anormaux (exp. VI), soit avec des $\delta^{*} \sigma^{*}$ " normal overlaps" issus du croisement de deux anormaux (exp. VII $b$ ) :

expérience VI : 3 An $x$ ㅇ An ;

expérience VII $b$ : $\approx \mathrm{F}_{\mathbf{1}} \mathrm{Nov}$ issu du VI $\times$ \& An.

Série III, comprend les croisements effectués entre of " normal overlaps " de la première génération et $\hat{o}^{\star}{ }^{\star}$ anormaux ou $\hat{o}^{\star}{ }^{*}$ eux-mêmes " normal overlaps ", c'est-à-dire phénotypiquement normaux mais se comportant génotypiquement comme des anormaux :

expérience VII $a:{ }^{*} \mathrm{An} \times$ o $\mathrm{F}_{\mathbf{1}}$ Nov issue du VI ;

expérience VIII : $\delta \mathrm{F}_{1}$ Nov issu du VI $\times$ o $\mathrm{F}_{1}$ Nov issue du VI. ration :

Série $I V$, ici les croisements sont effectués entre descendants "normal everlaps " de la $2^{\boldsymbol{\theta}}$ géné-

expériences IX : o $\mathrm{F}_{2} \mathrm{Nov}$ issu du VIII $\times \underset{\sim}{\circ} \mathrm{F}_{2} \mathrm{Nov}$ issue du VIII.

Mode d'élevage. Dans notre élevage, les femelles destinées à la reproduction sont séparées des mâles de la même portée à l'âge de I mois, moment où elles sont utilisées pour les différents croisements. Les primipares sont donc, le plus souvent, des souris très jeunes (âgées de 2 à 3 mois). Il nous a paru intéressant de voir si les résultats seraient les mêmes avec des primipares plus âgées, de façon à établir avec précision si c'est l'influence de l'âge de la mère ou celle du rang de la parturition qui agit sur la fréquence d'apparition de l'anomalie. Un certain nombre de femelles ont fourni jusqu'à 5 portées.

Pour l'étude de l'influence du rang de la parturition, le matériel a été réparti en 3 groupes : I) ${ }_{1}^{\text {re }}$ portée, primipares ; 2) $2^{\mathbf{e}}$ portée, bipares ; 3$) 3^{\mathbf{e}}, 4^{\mathbf{e}}$ et $5^{\mathbf{e}}$ portées, multipares, ces dernières groupées ensemble parce qu'ici les résultats sont sensiblement les mêmes.

Pour l'étude de l'influence du facteur saison, nous avons réparti le matériel en 4 trimestres, correspondant à peu près aux 4 saisons de l'année, tout en respectant la subdivision entre les primipares, bipares et multipares : septembre à novembre, décembre à février, mars à mai et juin à août.

Pour l'étude de l'influence de l'âge de la mère au moment de la première ou de la deuxième parturition, nous avons réparti les femelles en 5 groupes suivant leur âge et nous avons comparé les pourcentages de sujets atteints dans les différentes séries de croisements.

Enfin, pour faire ressortir la relation qui existe entre l'âge de la mère et le facteur saison, nous avons pris les mêmes 5 groupes suivant l'âge de la mère et avons étudié les résultats obtenus en divisant l'année en deux semestres : septembre à février et mars à août.

\section{RÉSULTATS}

Les résultats des 4 séries de croisements montrent que la fréquence d'apparition de sujets atteints d'hypotrichose périodique dépend, non seulement de l'âge de la mère au moment de la naissance, mais encore d'une part, du rang de parturition et d'autre part, de la saison à laquelle la naissance a lieu.

Pour l'étude de ces facteurs, nous avons retenu 256 I souris, dont 2 2I4 sujets normaux et 347 atteints d'hypotrichose périodique.

\section{Rang de la parturition}

Dans les croisements de la série I, on constate que seules les primipares fournissent 2,6 p. Ioo de descendants atteints. Les femelles atteintes d'hypotrichose périodique (croisements de la série II), fournissent un taux de sujets atteints plus 
TABLEAU I

Infuence du rang de la parturition

sur la fréquence d'apparition de l'hypotrichose périodique.

\begin{tabular}{|c|c|c|c|c|c|c|c|c|c|c|c|c|c|}
\hline \multirow{2}{*}{$\begin{array}{l}\text { Nu- } \\
\text { méro } \\
\text { de la } \\
\text { série }\end{array}$} & \multirow{2}{*}{$\begin{array}{l}\text { Parents } \\
0 \times q\end{array}$} & \multicolumn{4}{|c|}{ Primipares } & \multicolumn{4}{|c|}{ Bipares } & \multicolumn{3}{|c|}{$\begin{array}{l}\text { Multipares } \\
3^{\mathrm{e}}, 4^{\mathrm{e}}, 5^{\mathrm{e}} \\
\text { portées }\end{array}$} & \multirow{2}{*}{ 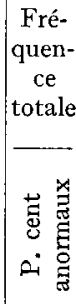 } \\
\hline & & 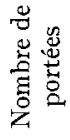 & $\stackrel{\vec{E}}{0}$ & 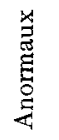 & 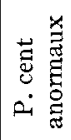 & 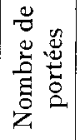 & 票 & 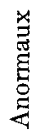 & 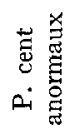 & 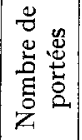 & 营 & 崫 & \\
\hline I & $\begin{array}{l}\text { II An } \times F_{1} N \text { du I } \\
\text { III } A n \times F_{6} N \text { du II } \\
\text { IV } F_{1} N \text { du } \times F_{1} N d u I\end{array}$ & 79 & 579 & 15 & 2,6 & 42 & 319 & - & 0 & 20 & 67 & - & 1,4 \\
\hline II & 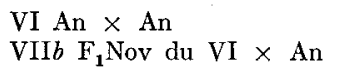 & 54 & 302 & 126 & 41,7 & 19 & 165 & 34 & 20,6 & 16 & 104 & - & 28 \\
\hline III & 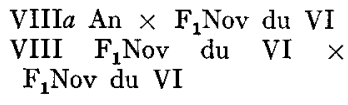 & 67 & 388 & 119 & 30,6 & 29 & 16 & 21 & 12,7 & 6 & 43 & - & 23,5 \\
\hline IV & $\begin{array}{l}\text { IX } F_{2} \text { Nov du VIII } \times \\
F_{2} \text { Nov du VIII }\end{array}$ & 45 & 287 & 32 & 11,1 & 4 & 34 & - & 0 & 1 & 8 & - & 9,7 \\
\hline
\end{tabular}

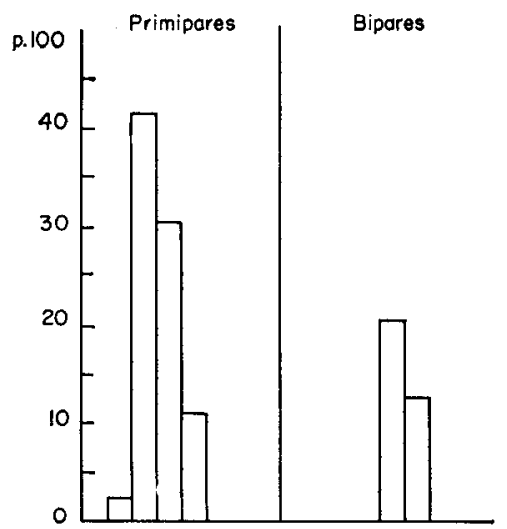

FIG. I. - Influence du rang de la parturition sur la fréquence d'apparition de l'hypotrichose périodique.

Série I : croisements des expériences II, III, IV.

Série II : croisements des expériences VI et VII $b$.

Série III : croisements des expériences VII $a$ et VIII.

Série IV : croisements des expériences IX. 
élevé que les mères "normal overlaps " de la première génération (croisements de la série III), 4I,7 contre 30,6 p. IOo. Il en est de même pour les bipares (croisements des séries II et III), 20,6 p. Ioo contre I2,7 p. Ioo. Enfin, dans les croisements de la série IV (femelles "normal overlaps) de la $2^{\mathrm{e}}$ génération), comme dans les croisements de la série I, seules les primipares fournissent des sujets atteints d'hypotrichose périodique (II, I p. IOO).

La fréquence d'apparition de l'anomalie la plus élevée a donc été observée chez les primipares, quel que soit le mode de croisements effectué ('Tabl. I, Fig. I). Dans les portées des bipares, on observe aussi des sujets anormaux, mais en nombre nettement moins grand, par contre aucun sujet anormal n'a été fourni par les multipares.

\section{Facteur saison}

Les expériences de croisements se poursuivant régulièrement tout au long de l'année, nous avons cherché à savoir si le facteur saison avait une influence sur la fréquence d'apparition de l'hypotrichose périodique. Nous avons comparé le pour-

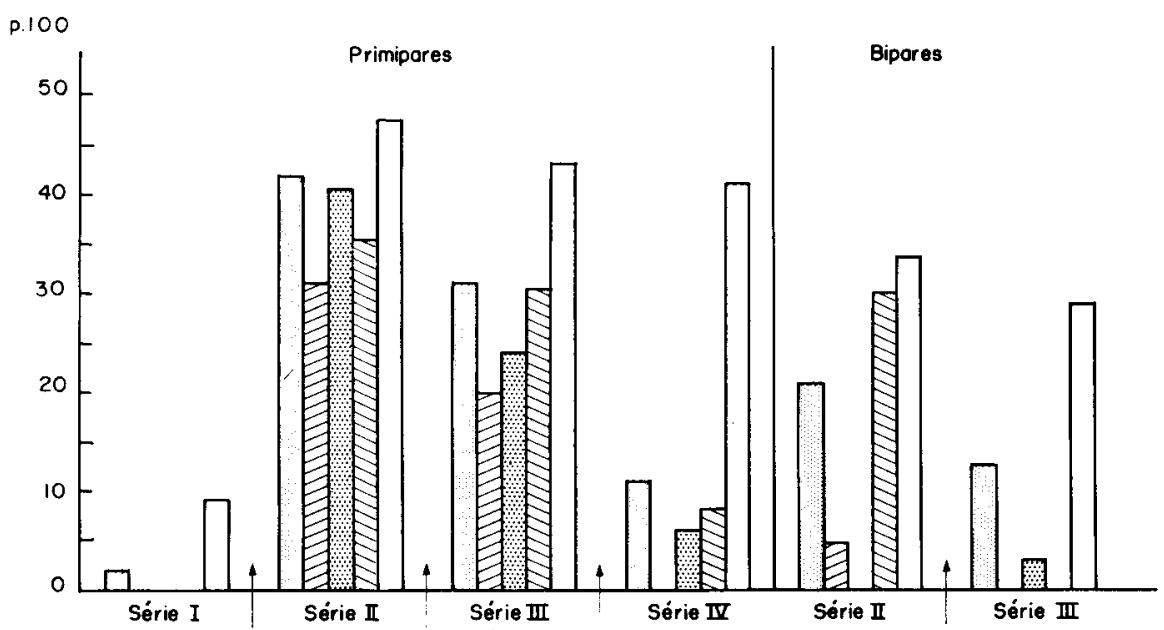

FIG. 2. - Influence du facteur saison sur la fréquence d'apparition de l'hypotrichose périodique.

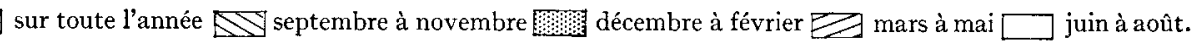

centage de fréquence d'apparition de sujets atteints dans les portées des primipares et des bipares, dans les différentes séries de croisements, suivant la saison de naissance de la portée (Tab1. 2 et Fig. 2).

Nous constatons que, quelle que soit la série de croisements effectués, la fréquence d'apparition de sujets atteints la plus grande se situe entre les mois de juin et d'août, et ceci, aussi bien chez les primipares que chez les bipares. Cependant, pour les primipares des croisements de la série II, le rôle de la saison semble moins accusé que pour celles des séries I, III et IV et que pour les bipares chez qui l'influence saisonnière est très frappante. 


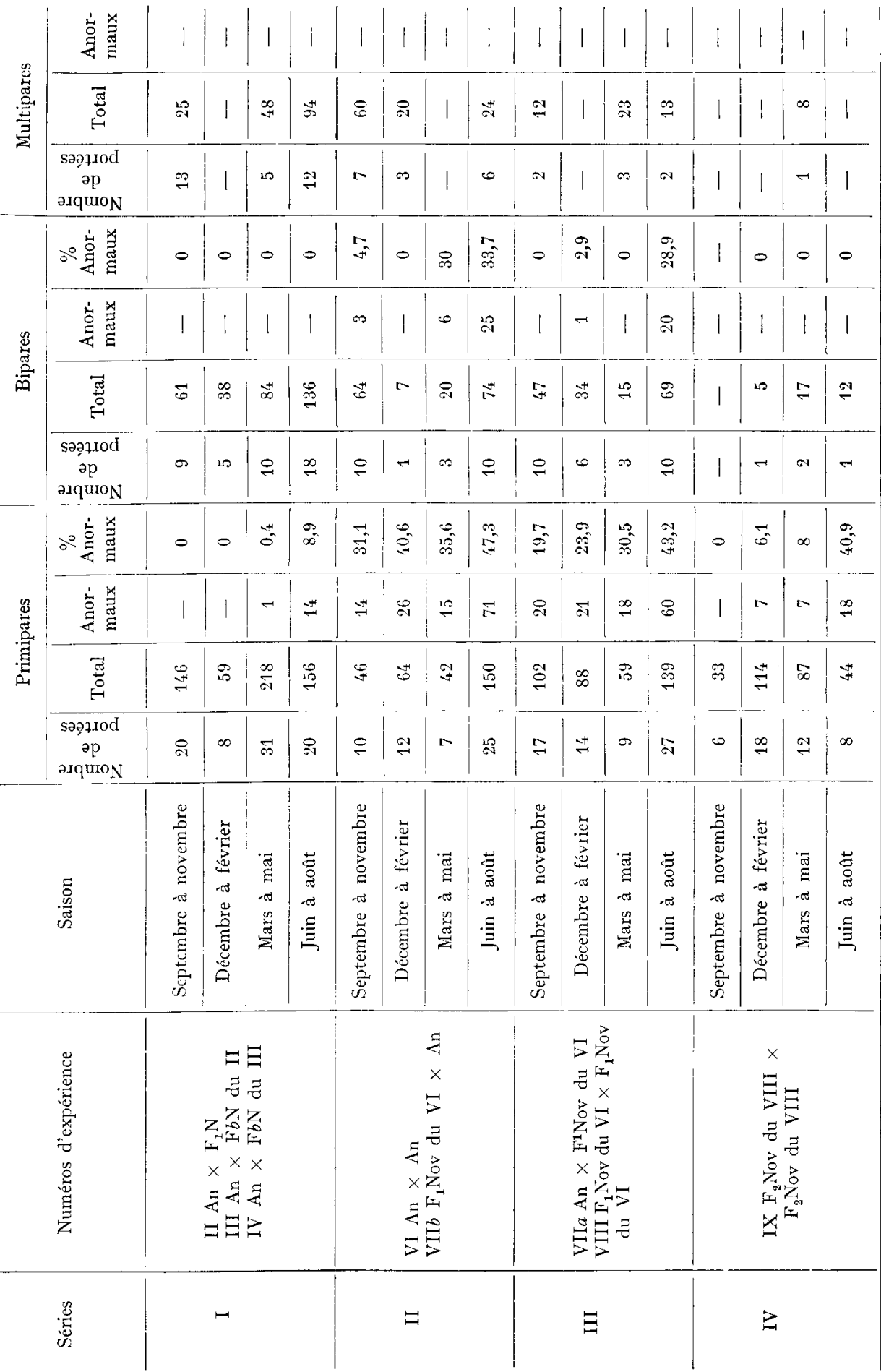




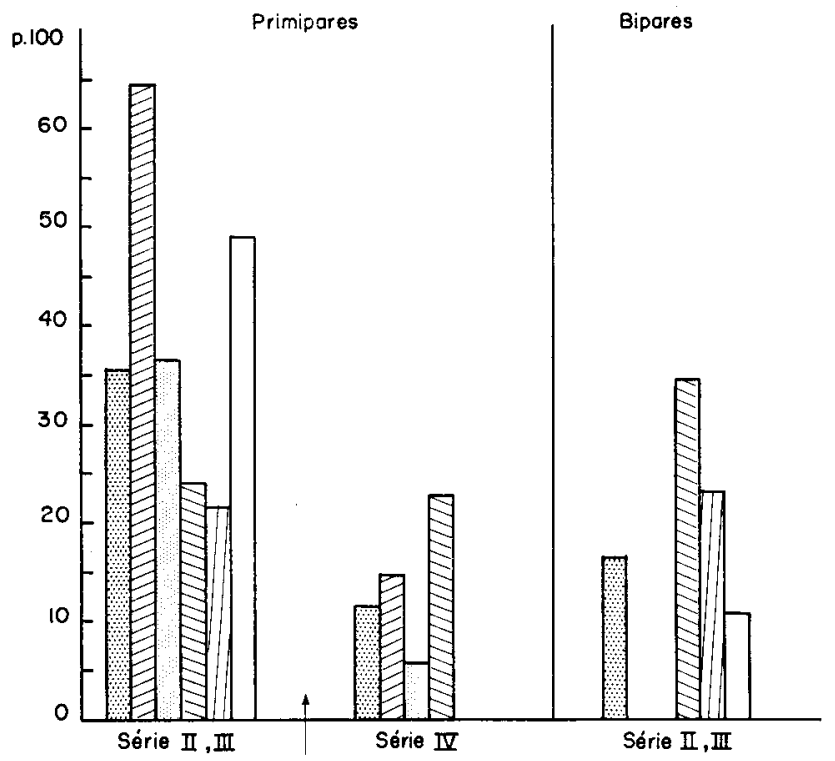

Frc. 3. - Infuence de l'âge de la mère sur la fréquence d'apparition de l'hypotrichose périodique.

Total

Eusqu'à to jours

De 7 I à 100 jours $\forall$ de ror à r 30 jours

[IIII de I 31 à 160 jours

$\square$ au-dessus de 16 I jours.

\section{TABI EAU 3}

Influence de l'âge de la mère et du rang de la parturition sur la fréquence d'apparition de l'hypotrichose périodique.

\begin{tabular}{|c|c|c|c|c|c|c|c|c|c|c|c|c|}
\hline \multirow{3}{*}{$\begin{array}{l}\text { Age de la mère } \\
\text { à la mise-bas }\end{array}$} & \multicolumn{4}{|c|}{$\begin{array}{l}\text { Séries II et III } \\
\text { expériences VI à VII }\end{array}$} & \multicolumn{4}{|c|}{$\begin{array}{c}\text { Série IV } \\
\text { expérience IX }\end{array}$} & \multicolumn{4}{|c|}{$\begin{array}{l}\text { Séries II et III } \\
\text { expériences VI à VIII }\end{array}$} \\
\hline & \multicolumn{8}{|c|}{ Primipares } & \multicolumn{4}{|c|}{ Bipares } \\
\hline & 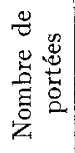 & $\stackrel{\vec{ت}}{\stackrel{7}{0}}$ & 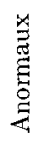 & ○。芯 & 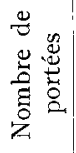 & 覃 & 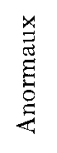 & 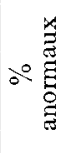 & 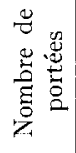 & 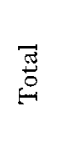 & 峞 & 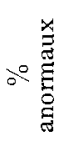 \\
\hline moins de 70 jours. & 15 & 94 & 61 & 64,8 & 6 & 42 & 6 & 14,3 & - & $\longrightarrow$ & 一 & \\
\hline De 71 à 100 jours & 48 & 265 & 96 & 36,2 & 19 & 125 & 7 & 5,6 & 1 & 5 & - & 0 \\
\hline De 101 à 130 jours & 28 & 195 & 47 & 24,1 & 14 & 85 & 19 & 22,3 & 9 & 55 & 19 & 34,5 \\
\hline De 131 à 160 jours & 17 & 93 & 20 & 21,5 & 3 & 19 & 一 & 0 & 13 & 69 & 16 & 23,1 \\
\hline Au-dessus de 161 jours & 8 & 43 & 21 & 48,8 & 1 & 10 & - & 0 & 29 & 196 & 20 & 10,2 \\
\hline
\end{tabular}




\section{Age de la mère et parturition}

Pour observer l'influence de l'âge de la mère sur la fréquence d'apparition de l'hypotrichose périodique, nous avons réparti les femelles en 5 groupes, suivant leur âge au moment de la première et de la deuxième mise bas, et nous avons comparé les pourcentages de sujets atteints dans les différentes séries de croisements (voir Tab1. 3 et Fig. 3).

TABLEAU 4

Infuence de l'âge de la mère et de la saison sur la fréquence d'apparition de l'hypotrichose périodique.

\begin{tabular}{|c|c|c|c|c|c|c|c|c|c|c|c|c|c|}
\hline \multirow{3}{*}{$\begin{array}{l}\text { Age de la mère } \\
\text { au moment } \\
\text { de la mise bas }\end{array}$} & \multirow{3}{*}{ Séries } & \multicolumn{6}{|c|}{ Primipares } & \multicolumn{6}{|c|}{ Bipares } \\
\hline & & \multicolumn{3}{|c|}{$\begin{array}{l}\text { Septembre à } \\
\text { février }\end{array}$} & \multicolumn{3}{|c|}{$\begin{array}{l}\text { Mars à } \\
\text { août }\end{array}$} & \multicolumn{3}{|c|}{$\begin{array}{l}\text { Septembre à } \\
\text { février }\end{array}$} & \multicolumn{3}{|c|}{$\begin{array}{c}\text { Mars à } \\
\text { août }\end{array}$} \\
\hline & & Total & $\begin{array}{l}\text { Anor- } \\
\text { maux }\end{array}$ & $\begin{array}{c}\% \\
\text { anor- } \\
\text { maux }\end{array}$ & Total & $\begin{array}{l}\text { Anor- } \\
\text { maux }\end{array}$ & $\begin{array}{c}\% \\
\text { anor- } \\
\text { maux }\end{array}$ & Total & $\begin{array}{l}\text { Anor- } \\
\text { maux }\end{array}$ & $\mid \begin{array}{c}\% \\
\text { anor- } \\
\text { maux }\end{array}$ & Total & $\begin{array}{l}\text { Anor- } \\
\text { maux }\end{array}$ & $\begin{array}{c}\% \\
\text { anor- } \\
\text { maux }\end{array}$ \\
\hline \multirow{2}{*}{$\begin{array}{l}\text { Moins de } \\
70 \text { jours }\end{array}$} & II, III & 41 & 30 & 73,1 & 53 & 31 & 58,4 & - & - & - & - & - & - \\
\hline & IV & 35 & 6 & 17,1 & 7 & - & 0 & $\ldots$ & - & - & - & - & - \\
\hline \multirow{2}{*}{$\begin{array}{l}\text { De } 71 \text { à } \\
100 \text { jours }\end{array}$} & II, III & 125 & 30 & 24 & 140 & 66 & 47,1 & 5 & - & 0 & - & - & - \\
\hline & IV & 73 & - & 0 & 72 & 18 & 22 & - & - & $-\ldots$ & - & - & - \\
\hline \multirow{2}{*}{$\begin{array}{l}\text { De } 101 \text { à } \\
130 \text { jours }\end{array}$} & II, III & 101 & 18 & 17,8 & 94 & 29 & 30,8 & 30 & 3 & 10 & 25 & 16 & 64 \\
\hline & IV & 21 & 1 & 4,7 & 72 & 18 & 22,2 & - & - & - & - & - & - \\
\hline \multirow{2}{*}{$\begin{array}{l}\text { De } 131 \text { à } \\
160 \text { jours }\end{array}$} & II, III & 18 & - & 0 & 75 & 20 & 26,6 & 37 & 1 & 2,7 & 32 & 15 & 46,8 \\
\hline & IV & 11 & - & 0 & - & - & - & - & - & - & - & - & - \\
\hline \multirow{2}{*}{$\begin{array}{l}\text { Au-dessus de } \\
161 \text { jours }\end{array}$} & II, III & 15 & 3 & 20 & 28 & 18 & 64 & 75 & - & - & 121 & 20 & 16,5 \\
\hline & IV & 13 & - & - & - & $\ldots$ & - & - & - & - & - & - & - \\
\hline
\end{tabular}

Nous constatons, dans les croisements des séries II et III, que les femelles es plus jeunes (jusqu'à 70 jours), fournissent le pourcentage de sujets atteints le plus élevé (64,8 p. roo), ce pourcentage diminue progressivement au fur et à mesure que l'âge de la mère augmente et ceci jusqu'à l'âge de I6o jours; plus tard, chez les femelles âgées (plus de I6I jours au moment de la première mise bas), on observe une nouvelle recrudescence de la fréquence d'apparition de sujets atteints (48,8 p. roo). 
Par contre, dans les croisements de la série IV (croisements entre géniteurs " normal overlaps " de la $2^{\mathrm{e}}$ génération), l'âge de la mère ne semble pas avoir la même influence sur la fréquence d'apparition de l'anomalie. Le pourcentage le plus élevé de sujets atteints (22,3 p. IOO) est constaté chez les femelles d'âge moyen (IOI à 130 jours), les plus jeunes fournissent un assez faible pourcentage de sujets anormatux ( $14,3 \mathrm{p}$. roo pour celles de moins de 70 jours et $5,6 \mathrm{p}$. Ioo pour celles de $7 \mathrm{I}$ à roo jours), et les femelles âgées de plus de I6I jours n'ont fourni aucun sujet atteint d'hypotrichose périodique.

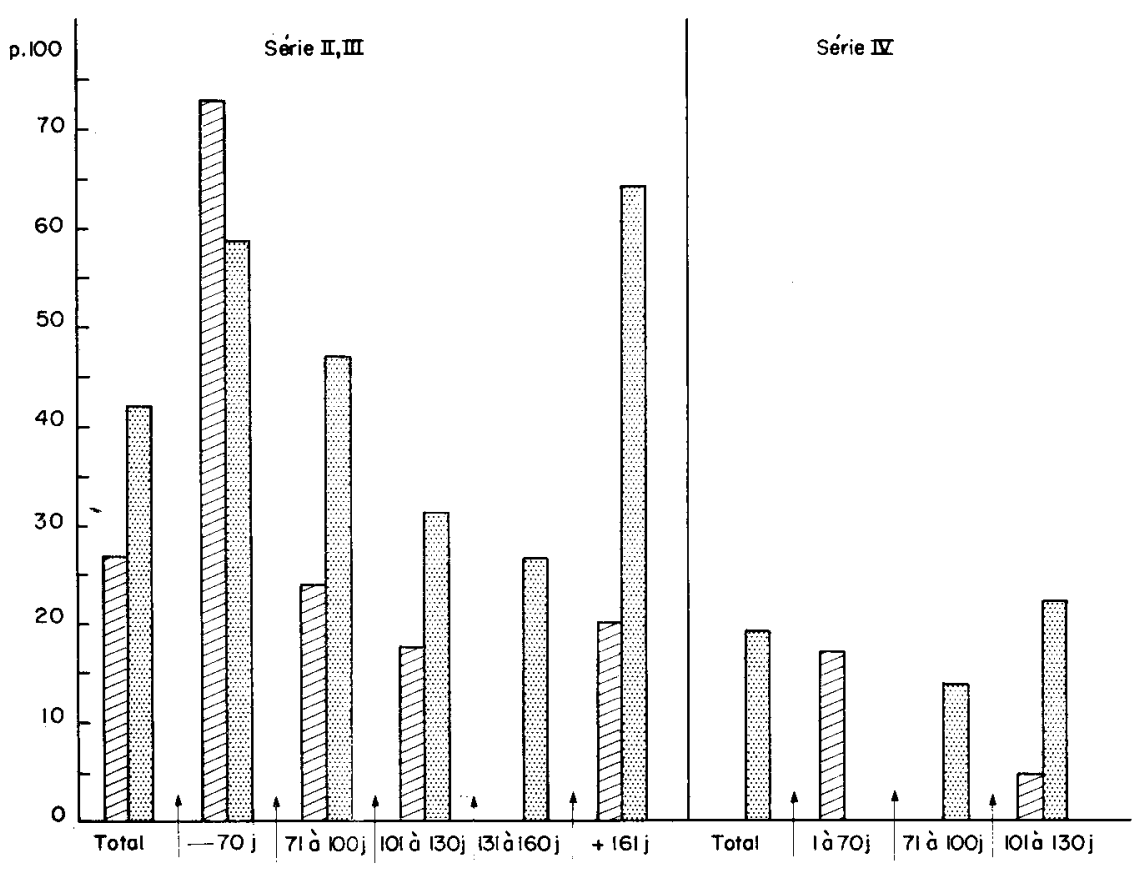

FIG. 4. - Influence de l'áge de la mère et de la saison sur la frequence d'apparition de l'hypotrichose périodique.

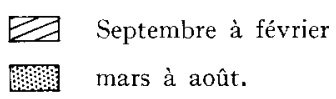

Parmi les bipares, dans les croisements des séries II et III, nous constatons que les mères d'âge moyen (IOI à I3O jours), au moment de la deuxième parturition, fournissent le pourcentage le plus élevé de sujets atteints (34,5 p. Ioo), pourcentage qui diminue progressivement au fur et à mesure que l'âge de la mère augmente pour ne donner que $10,2 \mathrm{p}$. Ioo de sujets atteints parmi les descendants des femelles âgées de plus de I6I jours. En comparant ces mères avec les primipares, on voit qu'elles se distinguent par le fait qu'on n'observe pas de recrudescence dans le nombre de sujets atteints chez les femelles les plus âgées (tabl. 3). 


\section{Age de la mère et facteur saison}

Dans l'étude des rapports existant entre l'influence de l'âge de la mère et celle du facteur saison, sur la fréquence d'apparition de l'hypotrichose périodique, quelle que soit la série de croisements effectués, nous constatons que les primipares très jeunes (moins de 70 jours), semblent échapper à 1'influence du facteur saison. Par contre, l'influence de ce facteur est de plus en plus nette au fur et à mesure que l'âge de la mère augmente. Il en est de même pour les bipares, c'est sur les femelles les plus âgées que le facteur saison a la plus grande influence sur la fréquence d'apparition de l'hypotrichose périodique. Le taux de sujets atteints nés entre les mois de mars et août est toujours plus important que celui des sujets nés entre septembre et février (tab1. 4 et Fig. 4).

\section{CONCLUSIONS}

De l'ensemble de cette étude, il ressort que les facteurs génétiques ne sont pas les seuls à avoir une influence sur la fréquence d'apparition de l'hypotrichose périodique chez la souris. D'autres facteurs jouent un rôle qu'il a été possible de mettre en évidence :

- le rang de parturition; la fréquence d'apparition de sujets anormaux la plus élevée est observée chez les primipares ;

- le facteur saison; c'est au cours de la période la plus chaude de l'année (juin à août), que la fréquence d'apparition de l'anomalie est la plus grande ;

- 1'âge de la mère ; ce sont les mères les plus jeunes qui fournissent le taux le plus élevé de descendants anormaux, puis viennent les mères les plus âgées et ce sont les femelles d'âge moyen qui en fournissent le moins.

Cette influence de 1'âge de la mère sur la fréquence de manifestation de différentes anomalies a été signalée dans la littérature à diverses reprises : WRIGHT, Kobozieff et Pomriaskinsky-Kobozieff, Holr, Green, Searle, Hauschka et Brown. Ces auteurs ont constaté, comme nous, que les mères less plus jeunes fournissent le taux le plus élevé de sujets anormaux.

Enfin, il semble y avoir une relation entre l'âge de la mère et le facteur saison. En effet, les mères très jeunes échappent à l'influence du facteur saison auquel les femelles d'âge intermédiaire et les femelles âgées sont particulièrement sensibles. Le taux de sujets anormaux le plus élevé est celui des animaux nés entre les mois de mars et août, de mères de plus de ror jours.

Reçu pour publication en décembre 1963 .

\section{SUMMARY}

PERIODICAL HYPOTRICHOSIS IN MICE. MATERNAL INFLUENCE : RANK OF PARTURITION SEASON FACTOR AND MOTHER'S AGE

Periodical hypotrichosis in mice is a recessive mutation, this recessivity is incomplete and of low penetrance. Other factors are also interfering in the frequency of abnormal subjects in the progeny. Here is an attempt to determine the part played by the maternal influence and the season 
factor. $256 \mathrm{I}$ mice $\mathrm{C}_{57} \mathrm{BL} / \mathrm{Jax} 6$ have been used, $22 \mathrm{I} 4$ normal and 347 mice showing periodical hypotrichosis.

Influence of the parturition rank : the highest rate of abnormal subjects is given by the primiparous, a lower rate by the biparous and no periodical hypotrichosis has been observed in the litters of multiparous mice.

The seasonal factor is important. The crossing experiments are made during the whole year but the highest rate of periodical hypotrichosis in the progeny has been noted between june and august.

Maternal age : we observed the highest rate of abnormal subjects in the litters of the youngest mothers (to 70 days), the rate is lower for the middle-aged mothers and gets up again with the eldest mothers (more than 161 days).

The influence of maternal age seems to be connected with the seasonal factor. We have noticed that the young primiparous are not affected by the season factor, whose influence is the more striking as the mother is older. The highest rate of abnormal subjects has been noticed between march and august. The phenomenon is a like in the biparous set. Therefore, the older the mother the more responsive she is to seasonal influence.

\section{RÉFÉRENCES BIBLIOGRAPHIQUES}

Bateman N., 1963. Selection and a maternal effect. Genetics Today, Proc. XIr Int. Congr. Genet., 1, 252.

Brumby P. J., I950. The infuence of maternal environment on growth in mice. Heredity, 14, I-I8.

Chai C. K., 1956. Body size inheritance in mice. Genetics, 41, 157-164.

DEOL M. S., 1955. Genetical studies on the skeleton of the mouse. XIV. Minor variations of the skull. J. Genel., 53, 498-514.

Falconer D. S., Ig60. The genetics of litter size in mice. J. Cell. Comp. Physiol., 56, suppl. I, I53-167.

Green E. L., Green M. C., r959. Transplantation of ova in mice. An attempt to modify the number of presacral vertebrae. J. Hered., 50, Iog-II4.

GREeN E. L., Russell W. L., 1951. A difference in skeletal type between reciprocal hybrids of two inbred strains of mice $\left(\mathrm{C}_{57} \mathrm{BLK}\right.$ and $\mathrm{C}_{3} \mathrm{H}$ ). Genetics, 36, 64I-6 $5 \mathrm{I}$.

HAUSCHKA T.S., BRowN E., 1954. Influence of maternal age on penetrance of "eyelids open " in newborn mice. Ann. N. Y. Acad. Sci., 57, 465-475.

HoLT S. B, 1948. The effect of maternal age on the manifestations of a polydactyl gene in mice. Ann. Eugen., 14, I 44-157.

KINDRED B. M, I962. A correlative response mediated through a maternal effect in the house mouse. Austr. J. Biol. Sci., 15, 352-361.

Kobozieff N., Pomriaskinsky-Kobozieff N. A., i946. Sur l'anomalie de l'oreille externe chez la souris. Influence de l'âge de la mère sur la fréquence d'apparition de l'anomalie. C. R. Acad. Sci., 222, roi 5-1or6.

Kobozieff N., Pomriaskinsky-Kobozieff N. A., Gemahling E., ig63. Hypotrichose périodique chez la souris. Ann. Biol. anim. Bioch. Biophys., 3, 207-218.

Kobozieff N., Pomriaskinsky-Kobozieff N. A., 1963. Periodical Hypotrichosis in Mice. Genetics Today, Proc. X1th Inst. Congr. Genet., 1, i85.

Maclaren A., Michie D., 1958. Factors affecting vertebral variation in mice. 4. Experimental proof of uterine basis of a maternal effect. J. Embr. Exp. Morph., 6, 645-659.

Penrose L. S., 1939. Maternal age, order of birth and developmental abnormalities. J. Ment. Sci., 85, I I 41-1 I5०.

REED S. C., 1936. Harelip in the house mouse. I. Effects of internal and external environments. Genetics, 21, $339-360$.

RusSEl. W. L., I948. Maternal influence on the number of lumbar vertebrae in mice raised from transplanted ovaries. Genetics, 33, 627-628.

SEARLE A. G., 1954. The influence of maternal age on development of the skeleton of the mouse. Ann. N. Y. Acad. Sci., 5\%, 558-563.

VENGE O., 1950. Studies of the maternal influence on the birth weight of rabbits. Acta Zool., 31, I-I48.

WRIGHT S., I934. An analysis of variability in number of digits in an inbred strain of guinea-pigs. Genetics 19. $506-536$. 